ARTIGO

Recebido em: $20 / 12 / 2017$

Aceito em: 20/04/2018

\title{
Desenvolvimento de Ontologia Ciente de Qualidade de Informações para o Domínio de Gerenciamento de Emergências
}

\author{
Development of Information Quality Aware Ontology for the \\ Improvement of Situational Awareness in the Emergency \\ Management Domain
}

\author{
Jordana Nogueira SILVA (jordana.nogueira@univem.edu.br)* \\ Allan César Moreira de OLIVEIRA (allan_oliveira@univem.edu.br)* \\ Gustavo Marttos Cáceres PEREIRA (gustavo.marttos@unesp.br) * \\ Valdir Amancio Pereira JUNIOR (valdir.a.junior@gmail.com)* \\ Lucas Zanco LADEIRA (lucaszl@lrc.ic.unicamp.br) * \\ Maria de Fátima TAVARES (fatimatavares@ibict.br) ${ }^{* *}$ \\ Leonardo Castro BOTEGA (leobotega@gmail.com)* \\ Seiji ISOTANI (sisotani@gmail.com) ${ }^{* *}$ \\ ${ }^{*}$ Grupo de Interação Humano-Computador, Centro Universitário Eurípides de Marília, Marília - SP. \\ ** Instituto Brasileiro de Informação em Ciência e Tecnologia, Brasília - DF. \\ *** Instituto de Ciências Matemáticas e Computação da Universidade de São Paulo - USP.
}

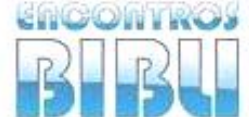

v. 23, n. $53,2018$. p. $184-200$

ISSN 1518-2924

\section{Resumo}

Para a comunidade de avaliação e gerenciamento de situações de emergências, determinar um processo de análise informacional para o desenvolvimento da Consciência da Situação (SAW) é um desafio, devido à limitada qualidade e complexidade dos dados, além da dinamicidade de ambientes reais monitorados. Operadores de sistemas de avaliação de situações de emergência, expostos a dados de qualidade incerta, estão suscetíveis a erros de SAW, diminuindo suas chances de uma tomada de decisão assertiva. Neste contexto, representar corretamente informações sobre tais situações, à medida que são coletadas e processadas, vai contribuir para melhor orientar humanos e sistemas sobre o que de fato acontece num ambiente de interesse. 0 presente trabalho visa contribuir com o problema de produção de melhores insumos na avaliação de situações de incêndios florestais, no âmbito do projeto DF100Fogo, apresentando o desenvolvimento de uma ontologia que agrega informações sobre a qualidade dos dados, promovendo ao operador de sistemas de emergência, uma melhor representação de dados sobre os incêndios. Um estudo de caso envolvendo um atendimento a uma situação de incêndio florestal será mostrado para demonstrar a aplicabilidade da ontologia junto à gestão da qualidade. Resultados apresentam um modelo de representação semântica, capaz de receber instâncias extraídas de dados de inteligência humana, com origem em sensoriamento participativo, e avaliadas quantitativamente sob aspectos de qualidade da informação.

Palavras-chave: Ontologias. Consciência Situacional. Qualidade de Dados e Informações. Gerenciamento de Emergências.

\section{Abstract}

One of the challenges approached by the community of emergency situation assessment and management is to determine a process of informational analysis to assist operators in developing Situational Awareness (SAW). Due to the complexity in data and uncertainty in its quality, besides the dynamicity in monitored environments, operators are susceptible to errors in their SAW, decreasing the chance of an assertive decision-making. In this context, correctly representing information about the situation, while they are gathered and processed, will contribute to better orient operators and systems regarding the ongoing situation. To this end, the goal of this paper is to contribute to the production of enhanced inputs in the situation assessment of forest fires for the DFWithoutFire project. More specifically, this paper presents the development of an ontology that aggregates information on 
data quality, promoting an improved representation of data about fires. A study case of a forest fire emergency response is showed to demonstrate the applicability of the ontology with the quality management. Results present a semantic representation model that is capable of receiving instances extracted from human intelligence data that originated from crowdsourcing and evaluated quantitatively on aspects of data quality.

Keywords: Ontologies. Situational Awareness. Data and Information Quality. Emergency Management.

\section{INTRODUÇÃo}

Nos últimos 15 anos o total de área queimada no Brasil chegou a $12.257 .764 \mathrm{~km}^{2}$ (INPE, 2017), sendo que uma das grandes preocupações é emissão de dióxido de carbono $\left(\mathrm{CO}^{2}\right)$, e sua contribuição para o agravamento do efeito estufa, consequentemente causando danos a camada de ozônio e alterações no clima. Outro dano causando pelas queimadas é a perda de espécie da fauna e flora, gerando um ambiente menos saudável e estável para vida humana. No ano de 2016 houve $1.023 .533 \mathrm{~km}^{2}$ de área queimada em todo território brasileiro.

Na área do bioma do Cerrado, localizado na região do Distrito Federal há grande recorrência de incêndios mesmo em áreas de preservação, e isso tem gerado uma preocupação ao setor público, que a cada ano acaba mobilizando mais recursos e esforços em operações de prevenção e combate a esses incêndios. Apesar de existir um Plano de Prevenção e Combate aos Incêndios Florestais ${ }^{1}$, o Distrito Federal ainda não possui um sistema próprio para o gerenciamento de incêndios florestais, fazendo uso atualmente do Sisfogo $^{2}$ (Sistema Nacional de Informações de Incêndios) do IBAMA (Instituto Brasileiro do Meio Ambiente e Recursos Naturais Renováveis). Dentro deste contexto o presente trabalho visa desenvolver recursos de representação de dados para o DF100Fogo, sistema que tem seu desenvolvimento e manutenção sob responsabilidade do Centro Universitário Eurípides de Marília (UNIVEM), Instituto Brasileiro de Informação em Ciência e Tecnologia (IBICT) e Universidade Federal de São Carlos (UFSCar).

Tal sistema, tem por objetivo auxiliar o Corpo de Bombeiros do Distrito Federal e as Brigadas do Jardim Botânico de Brasília (JBB) no combate e controle de incêndios florestais. Segundo a Secretaria de Estado do Meio Ambiente (SEMA), o JBB é composto por uma área de bioma de Cerrado com cerca de 500 hectares e possuindo assim um maior endemismo (fauna e flora) e diversidade de espécies, além de ser considerado o berço das águas pois abriga as três principais nascentes das bacias da América do Sul. O SEMA também cita que o cerrado está entre as 25 áreas classificadas como críticas para a conservação da biodiversidade biológica no mundo.

\section{MOTIVAÇÃO E OBJETIVOS}

A Consciência da Situação (SAW), de operadores que trabalham no domínio de gerenciamento de emergências torna-se um fator crucial na aquisição de melhores subsídios informacionais, sustentando assim todo processo de tomada de decisão desses operadores. Adquirir e manter a SAW de humanos operadores de sistemas críticos como os de gerenciamento de emergências em incêndios florestais, transforma-se em uma tarefa complexa. A dinamicidade do ambiente presente, o grande volume de dados e as muitas variáveis presentes no ambiente real, impactam negativamente no desenvolvimento e aquisição da SAW por tal operadores.

A produção de incerteza para a construção de SAW nesse domínio, também pode ser consequência dos dados provenientes principalmente de relatos humanos (HUMINT) que em geral são heterogêneos, imprevisíveis, complexos e dinâmicos. Esse tipo de contexto associado a limitados métodos para a representação de qualidade de informações, cria um ambiente totalmente inapropriado para que operadores responsáveis por tomar decisões de impacto, consigam atingir e desenvolver os três níveis de SAW, percepção, compreensão e projeção.

O objetivo geral desse trabalho é contribuir com o desenvolvimento da SAW de operadores de sistemas de gerenciamento de emergência, através do uso de ontologias para uma representação de dados mais clara e objetiva, mostrando ao operador humano todas as

\footnotetext{
10 Plano de Prevenção e Combate aos Incêndios Florestais do Distrito Federal foi instituído pelo Decreto no 17.431, de 11 de junho de 1996.

2 Sisfogo - http://www.ibama.gov.br/sistemas/sisfogo
} 
possíveis relações, entidades e seus atributos dentro de uma situação de emergência com incêndio. Especificamente 0 presente trabalho objetiva apresentar o desenvolvimento de uma ontologia no domínio de incêndios florestais, que faça uso de uma metodologia de gestão de qualidade para qualificar e quantificar os dados utilizados pela mesma, auxiliando assim sistemas e humanos operados em processos de inferência sobre a situação de emergência com os incêndios.

Para se cumprir o objetivo proposto, a metodologia empregada se apoia nas seguintes atividades: (1) Pesquisa sobre os principais temas presentes nesse trabalho como gerenciamento de emergência, consciência situacional, gerenciamento de emergência com incêndios; (2) Desenvolvimento e aplicação de um questionário à especialistas do domínio; (3) Aplicação da técnica de Análise de Tarefas Dirigidas por Objetivos (Goal-Directed Task Analysis - GDTA); (4) Utilização da metodologia de Noy e McGuinness (2001) e do vocabulário DQV (Data Quality Vocabulary), para o desenvolvimento da ontologia. (5) Utilização da metodologia IQESA (Information Quality Assessment Methodology in the Context of Emergency Situation Awareness), para aplicação de métricas ${ }^{3}$ e dimensões ${ }^{4}$ de qualidade de dados. (6) Metodologia exploratória dirigida por estudo de caso, validando a ontologia desenvolvida e executando testes através de consultas SPARQL.

\section{TRABALHOS RELACIONADOS}

O primeiro trabalho correlato ao desenvolvido por esse artigo foi de Li et al. (2008), onde em seu artigo "Building a Practical Ontology for Emergency Response Systems" foi proposto o desenvolvimento de uma ontologia de topo que pode ser aplicada a diversas emergências, pois ela utiliza conceitos semânticos padronizados.

Para a utilização dessa ontologia foi necessário seguir quatro etapas, sendo elas: (1) Preparação da resposta: antes de responder a emergência é necessário verificar sua veracidade, e após isso fazer uso de base de dados anteriores para escolha de uma melhor solução. (2) Resposta a emergência: nessa etapa é enviado uma equipe ao local da emergência e estabelecida uma comunicação confiável com a base, caso seja necessário é possível consultar um grupo de peritos para situações difíceis e imprevistas. (3) Resgate em emergência: essa etapa é feita caso haja vítimas a serem resgatas e atendidas, todo o monitoramento a emergência é feito em tempo real, e caso haja alterações no cenário, os passos anteriores devem ser refeitos. (4) Manipulação de resíduos: nessa etapa uma investigação é feita a fim de identificar as causas do incidente, após essa apuração, é feita a limpeza e restauração do local onde ocorreu o incidente e prestado apoio social as vítimas. Essa ontologia usa conceitos genéricos de uma situação de emergência e conta com um vocabulário comum para o domínio. Tal conceito pode se torna um desafio caso haja alguma situação imprevista sem abrangência por esses termos. Segundo o artigo os testes realizados foram feitos em ambiente controlado o que dificulta saber o real potencial dessa ontologia, e atualmente ela ainda não faz uso de metodologias para a gestão de qualidade de dados e informações.

O segundo trabalho relacionado encontrado foi o de Amailef \& Lu (2013), tais autores desenvolveram um sistema chamado OS-CBR (Ontology-Supported Case-Based Reasoning), utilizando a metodologia de raciocínio baseado em casos junto com recursos da ontologia. Os autores descrevem em seu artigo os benefícios de juntar o CBR (Case-Based Resoning) com a tecnologia ontológica, melhorando assim a eficiência dos tomadores de decisão em uma situação de emergência. 0 sistema conta com medidas de similaridades baseadas em ontologias, evitando assim problemas de sinônimos nos dados. Outro benefício apresentado pelos autores é a capacidade que o sistema possui de aprender com situações do passado, visando assim gerar melhores solução para presentes situações de emergências. Esse sistema foi baseado em dispositivos móveis (MERS - Mobile-Based Emergency Response System) e segundo os autores esse tipo de abordagem é significativa tanto na teoria como na prática.

Os testes foram realizados no domínio de localização dos desastres (espacial e temporal), e os dados para esses testes de usabilidade da abordagem proposta, foram

\footnotetext{
${ }^{3}$ Forma de inferir, concluir ou avaliar cada dimensão, composta por variáveis arranjadas de forma objetiva e subjetiva.

4 Perspectiva sob a qual busca-se medir e analisar a qualidade.
} 
fornecidos pelos próprios autores. Também não foi identificado o uso de metodologias para a gestão de qualidade de dados e informações, e o segundo o artigo o trabalho ainda se encontra em desenvolvimento, sendo os próximos passos a realização de um estudo mais aprofundado em outras sessões do MERS e o desenvolvimento de mecanismos que facilitem a integração de soluções MERS com sistemas de emergências existentes baseados em informações provenientes da internet.

Outro trabalho correlato encontrado, foi a ontologia Fire, de Souza et al. (2014), em seu artigo "An Application of geographical and Statistical Linked Data to Ecology: The Brazilian Cerrado Ontology Network and Qualitative Reasoning Models" (2014) ele propõe o desenvolvimento de uma ontologia para representar o conhecimento científico sobre ecologias da vegetação presente no Bioma do Cerrado Brasileiro. Em seu trabalho Souza (2014), faz uso de várias ontologias de diferentes domínios como o de estatística, clima, tempo e incêndio, para compor sua ontologia. A ontologia Fire tem por objetivo representar os diferentes modos e eventos de incêndios, e os conceitos sobre incêndios que ocorrem na vegetação natural suas características, causas e efeitos, com foco no domínio da vegetação Cerrado (Souza et al., 2014).

Segundo o artigo os testes de validação desta ontologia foram feitos em dados abertos das agências do governo brasileiro, como INMET (Instituto Nacional de Meteorologia do Brasil), INPE (Instituto Nacional de Pesquisa Espacial), IBGE (Instituto Brasileiro de Geografia e Estatística) e LAPIG - UFG (Processamento de Imagem e Laboratório de Geoprocessamento da Universidade Federal de Goiás). 0 artigo também informa que esses resultados são um primeiro passo na associação entre conjuntos de dados e modelos qualitativos, e para próximos trabalhos é esperado mapear dados numéricos em pontos e intervalos relevantes, estabelecendo uma correspondência entre dados numéricos e valores qualitativos, além de produzir automaticamente uma interpretação do significado de influências diretas e proporcionalidades qualitativas, de modo que o modelo causal possa ser lido e os resultados da simulação interpretados para o usuário.

\section{O PROJETO DF100FOGO}

O projeto DF100Fogo tem o objetivo de auxiliar o Corpo de Bombeiros do Distrito Federal e as Brigadas do Jardim Botânico de Brasília (JBB), no combate e controle de incêndios florestais em áreas de conservação. 0 projeto é desenvolvimento e mantido pelo Centro Universitário Eurípides de Marília (UNIVEM) em parceria com o Instituto Brasileiro de Informação em Ciência e Tecnologia (IBICT) e a Universidade Federal de São Carlos (UFSCar). Tal projeto é composto por um aplicativo destinado à comunidade, que envia aos bombeiros alertas, em texto, foto ou áudio, sobre focos de incêndios em áreas urbanas e protegidas. Há também um aplicativo de uso de bombeiros e brigadistas que ficam em patrulha pelo JBB, e seu objetivo é manter a comunicação de maneira mais eficiente entre os bombeiros e brigadistas de campo com a base dos bombeiros. Com esse aplicativo, os bombeiros têm acesso a uma lista de notificações sobre incêndios e conseguem visualizar esses incêndios no mapa.

Na arquitetura do DF100Fogo há também um sistema servidor que faz a extração e processamento de todos os alertas, baseando-se nas técnicas de Fusão de Dados do modelo Quantify (Quality-Aware Human-Driven Information Fusion Model) (Botega et al., 2017). Junto a esse sistema há ainda um sistema administrador que permite visualizar informações sobre a real situação da emergência, possibilitando também agregar informações de georreferenciamento e clima à situação, através de serviços externos que são consumidos pelo sistema administrador do DF100Fogo.

\section{DESENVOLVIMENTO DE ONTOLOGIA CIENTE 5 DE QUALIDADE DE INFORMAÇÕES}

Ontologia teve seus estudos iniciais dentro da área filosófica, porém atualmente esse termo tem sido estudado por áreas como Ciência da Computação e Ciência da Informação. Uma definição bastante utilizada na literatura para definir ontologias é dada por Gruber (1993) onde ele diz que "uma ontologia é uma especificação explicita de uma conceitualização". Em tal definição podemos compreender que especificação explicita pode

50 termo "ciente" significa sabedor ou conhecedor de algo ou alguma coisa.

https://dicionariodoaurelio.com/ciente 
ser compreendida como uma representação de conceitos e relacionamentos dentro de um contexto. Dentro da Ciência da Informação podemos citar a definição de ontologia dada por Campos e Campos (2014), onde as autoras afirmam que as ontologias fornecem "[...] um modelo pra representar os pressupostos epistemológicos e ontológicos, relevantes para o entendimento de pesquisas e seu tratamento computacional através de iniciativas de dados interligados [...]".

Em ambas as áreas o foco no uso da ontologia se dá em permitir o uso de mecanismos computacionais que sejam capazes de compreender o sentido da informação. Essas ferramentas auxiliam na aproximação da linguagem humana com a linguagem computacional.

Após estudos sobre os principais temas presentes nesse trabalho, para se ter conhecimento sobre o domínio de incêndios, foi desenvolvido e aplicado um questionário ao Corpo de Bombeiros Militar do Distrito Federal (CBMDF) e às Brigadas de Combate a Incêndios do Jardim Botânico de Brasília. Com as respostas adquiridas, foi possível identificar quais informações são prioritárias para a tomada de decisão de operadores frente a uma situação de incêndio no JBB, e quais são os serviços e fontes de dados que devem ser consumidos para provê-las ou inferi-las. A Figura 1 demostra uma árvore de atributos criada a partir dos principais termos e entidades identificados nas repostas fornecidas pelos bombeiros, levando em consideração a patente e o tempo de serviço de cada bombeiro.

A árvore de atributos se torna um recurso de extrema importância para as demais fases da metodologia, pois é possível verificar uma hierarquia de atributos através dos nós que à compõe, e para a fase de qualificação dos dados ela fornece insumo para identificar os requisitos que devem ser quantificados e qualificados. 0 nó central da árvore de atributos é a situação de emergência com incêndio, e os nós que a ele estão ligados são as entidades que compõe essa situação, cada qual com seus atributos também chamados de folhas, esses atributos descrevem cada entidade.

Em um atendimento a uma solicitação de incêndio florestal, as principais entidades que devem ser identificadas são:

- Informações sobre o local em que está ocorrendo o incêndio; além de informações sobre vítimas, quantidade e estado;

- Qualificações sobre o solicitante ou denunciante;

- Informações sobre o fogo, se foco de incêndio é pequeno, médio ou grande;

- Informações sobre o clima na região do incêndio, a velocidade do vento, taxa de umidade, etc.;

- Tipo de vegetação atingida pelo incêndio e da topologia em que o incêndio está ocorrendo;

A partir desses resultados foi possível atualizar desenvolver a primeira versão de um modelo de análise de tarefas. Esse modelo representa além de objetivos a serem alcançados, decisões a serem tomadas, tarefas a serem cumpridas e requisitos informacionais para contemplar essa demanda. 0 nível de importância de cada informação revelada pela entrevista possibilitou criar uma escala de prioridades informacionais (Endsley, 2003).

\subsection{ANÁLISE DE TAREFAS DIRIGIDA POR OBJETIVOS (GDTA - GOAL-DIRECTED TASK ANALYSIS)}

O uso da metodologia GDTA (Endsley, 2003), tem como objetivo a identificação dos principais aspectos referente ao domínio que o mesmo está sendo aplicado. Em sistemas críticos, ele é usado para a identificação dos principais objetivos a serem alcançados com sucesso pelo operador, além de contribuir com a aquisição e desenvolvimento da SAW de tal operador. Endsley (2001), descreve a análise que o GDTA aplica como: “(...) este tipo de análise baseia-se em metas ou objetivos, e não em tarefas (como pode ser uma análise de tarefas tradicional). Isso ocorre porque os objetivos são a base para a tomada de decisões em muitos ambientes complexos". 


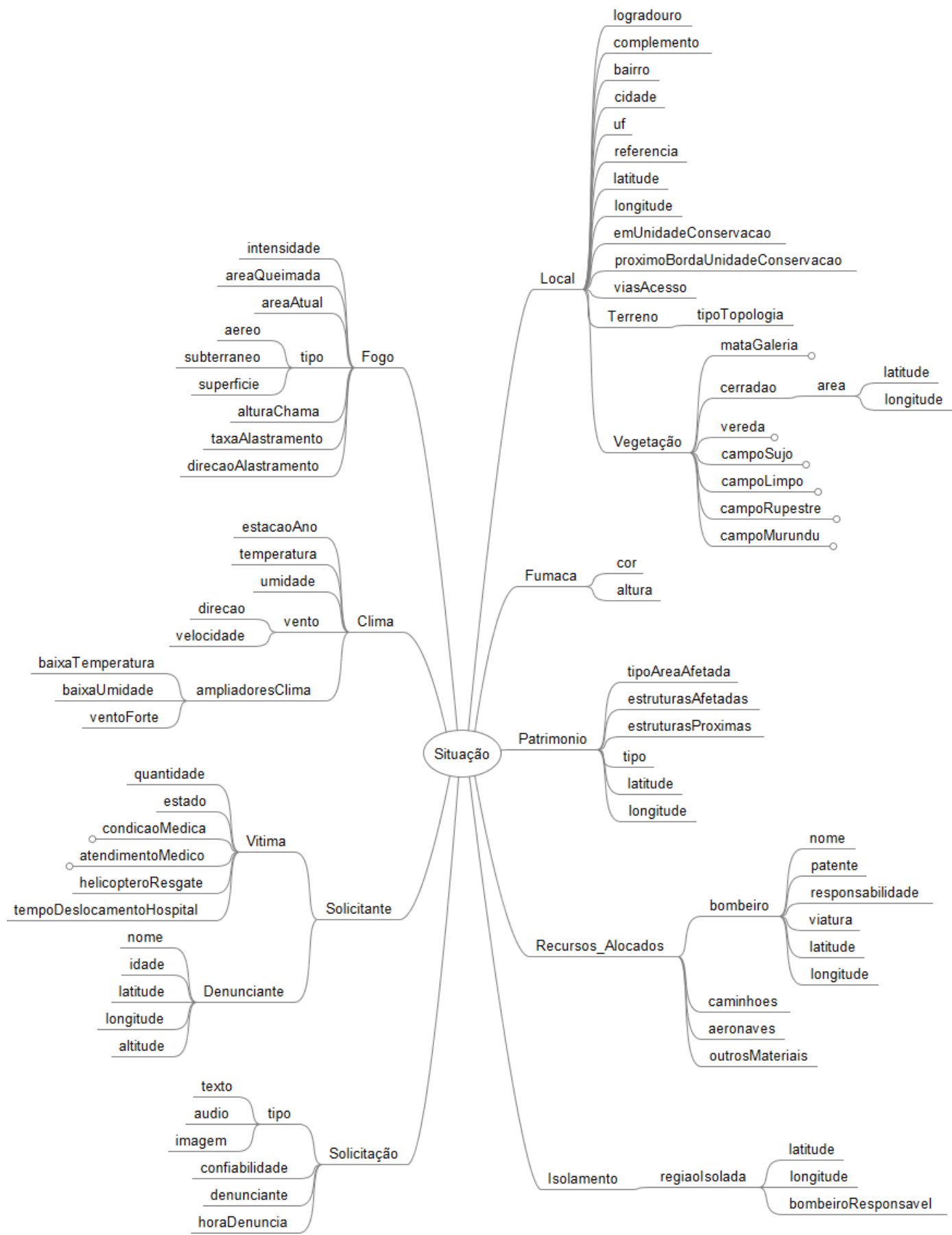

Figura 1: Árvore de atributos de emergências em incêndios florestais.

\subsection{CONSTRUÇÃO DE UMA ONTOLOGIA DE DOMÍNIO}

A metodologia adotada para a construção e o desenvolvimento da ontologia de domínio, será a metodologia 101 (Ontology Development 101) de Noy McGuinness (2001). Seguindo um ciclo de sete passos essa metodologia se tornou a mais utilizada em construção de ontologias devido a sua simplicidade em ser entendida e aplicada a qualquer domínio. Abaixo serão descritos todos os passos para o desenvolvimento da ontologia no presente trabalho.

- Definir o Escopo: A ontologia desenvolvida tem o domínio como sendo incêndios florestais, a mesma será usada para auxiliar os Bombeiros que atuam no JBB ao combate e controle de situações de incêndios.

- Considerar o Reuso: Após pesquisa feitas, foi localizado uma ontologia chamada Fire (Souza et al., 2014), que poderia ser reutilizada para o mesmo domínio, porém 
seu foco era o efeito nas estruturas e fisionomias que os incêndios no cerrado brasileiro causam, por isso essa ontologia não foi utilizada, porém foi estuda e considerada um trabalho correlato.

- Enumerar Termos: Essa etapa foi concluída após uma análise das respostas obtidas pelos bombeiros, sendo assim foi feito um levantamento dos principais termos citados por eles, levando em consideração a patente de cada bombeiro.

- Definir Classes e Propriedades: Havia no questionário algumas perguntas que revelavam quais seriam as informações prioritárias como por exemplo: A sétima pergunta era: Quais são os requisitos ou critérios ideais para atender a uma solicitação de incêndio? A resposta dessa pergunta foi o insumo necessário para a definição de classes e propriedades.

- Definir Restrições: Após a definição de classe e propriedades, já com todo conhecimento obtido sobre o domínio, as restrições ficam mais claras, sendo um dos exemplos: "incêndio acontece em um lugar" sendo essa restrição tratada pela ontologia como $1-\mathrm{N}$.

A Figura 2 demonstra a ontologia desenvolvida seguindo os passos da metodologia 101 conforme descrito acima.

\subsection{APLICAÇÃO DE MÉTRICAS E DIMENSÕES PARA QUALIFICAR E QUANTIFICAR OS DADOS}

Nessa etapa foram definidas as funções e métricas para quantificar as dimensões, utilizando a metodologia IQESA (Botega, 2016). Tal metodologia visa ilustrar todas as fases para avaliar e representar a qualidade dos dados e informações como parte de um processo de avaliação de informações, cujo objetivo é obter e fornecer a manutenção do SAW no contexto da gestão de emergências. A IQESA faz uso das dimensões Atualidade, Completude, Consistência, Relevância e Certeza para medir a qualidade de uma informação. Tais dimensões serão usadas devido à complexidade apresentada pelo domínio em que esse trabalho está sendo aplicado.

Ambientes complexos produzem dados imprecisos, incompletos e difusos. Tais problemas se agravam se esses dados forem provenientes de inteligência humana (Human Intelligence - HUMINT) que tendem a ser omissos, imprecisos e tendenciosos. Outro fator levado em consideração na escolha das dimensões é a dinamicidade das informações, o que leva a produção de incerteza por parte do operador do sistema na tomada de decisão. Nas próximas subseções tais dimensões serão detalhadas quanto a seu uso dentro deste trabalho. Junto à ontologia será utilizado o Vocabulário de Qualidade de Dados (Data Quality Vocabulary - DQV), que será responsável por identificar as relações das dimensões de qualidade com as instâncias da ontologia.

O DQV é um modelo de boas práticas para expressar a qualidade de dados. Foi desenvolvido pelo Grupo do W3C (World Wide Web Consortium), visando estimular, facilitar a publicação, intercambio e consumo de metadados de qualidade, para cada etapa do ciclo de vida de um conjunto de dados. 


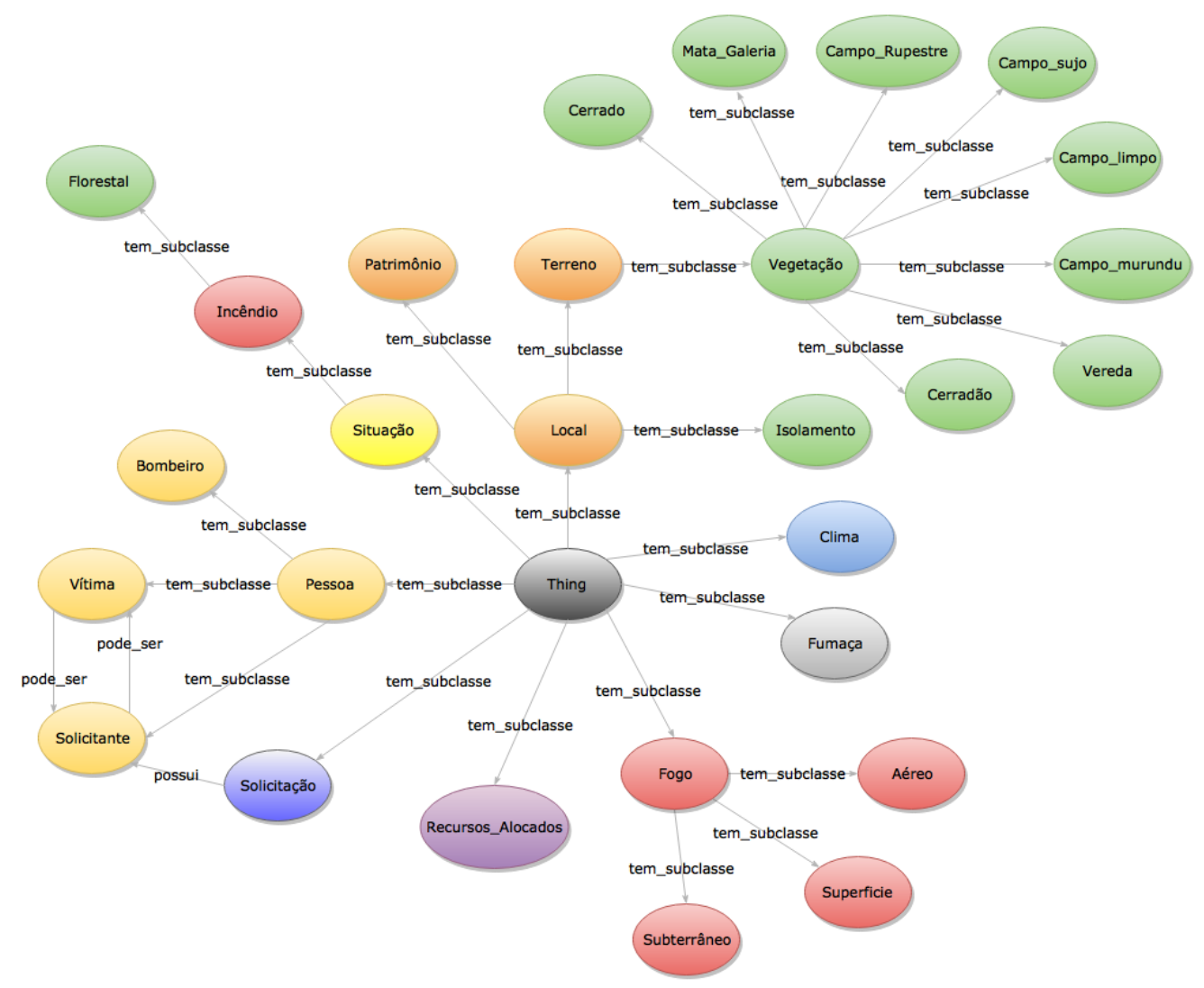

Figura 2: Ontologia DF100Fogo desenvolvida com a metodologia de Noy \& McGuinness (2001)

\subsubsection{AVALIAÇÃO DE ATUALIDADE}

A avaliação da Atualidade trata-se de um progresso temporal de um evento, sua contribuição para o desenvolvimento a SAW de operadores de sistemas de emergências é essencial. Se os dados temporais estiverem errados ou imprecisos, a situação pode ser interpretada de maneira incorreta, causando assim uma tomada de decisão errada por parte dos operadores (Botega, 2016).

Para o domínio de incêndio florestal, a sua métrica será obtida através de dois atributos presentes na situação de emergência: (1) hora que a solicitação/alerta foi gerada e a (2) hora atual do sistema. Essa avaliação irá resultar em dois valores: um indicador quantitativo para a existência dos dois atributos e os valores para calcular o tempo de atendimento a solicitação. A Fórmula 1 consiste em efetuar a subtração dos dois atributos, hora em que o alerta foi gerado e hora atual do sistema.

$$
T_{s}=\sum_{y=1}^{2}-\theta
$$

Onde $\theta$ consiste num índice composto pelo horário atual, subtraído dos seguintes atributos temporais: a hora do evento contida em relato, o momento que o relato foi processado e o tempo gasto para processar tais informações ao encontrar objetos e atributos.

\subsubsection{AVALIAÇÃO DE COMPLETUDE}

Para avaliar a completude no domínio de incêndio florestal, a arvore de atributos foi utilizada, pois a mesma foi construída com base nas respostas obtidas junto aos bombeiros 
no questionário aplicado, estando ali presente todos os atributos que possuem prioridade para se atender uma solicitação de incêndio pelos bombeiros.

$$
C=S\left[\left(\frac{\sum \beta * \gamma}{\sum \gamma} * 0,9\right)+0,1\right]
$$

Onde que $S$ representa a presença do objeto a ser avaliado, quando presente, $S=1 \mathrm{e}$ quando ausente, $S=0 ; \beta$ representa o atributo que descreve o objeto, quando presente é igual a 1 e quando ausente é 0 ; o $\gamma$ representa o peso, que, quando considerado prioritário, tem valor igual a 2 e quando não prioritário é recebe o valor igual 1. Para cada peso presente deve ser feita a somatória da multiplicação e o seu resultado dividido pelo total de atributos prioritários. 0 valor resultante desta fórmula deve variar entre 0 a 100\% (Melo, 2017).

\subsubsection{AVALIAÇÃO DE RELEVÂNCIA}

A dimensão de Relevância não possui fórmula para o seu cálculo, ela consiste em identificar os atributos presentes relevantes no objeto, atribuindo a eles o valor de 0 para os não relevantes ou 1 para os relevantes. Um exemplo para o cálculo da relevância pode ser demonstrado na classe Local presente na ontologia, onde dentro de um atendimento a uma emergência os valores complemento, referência, latitude, longitude possuem mais relevância que os valores de cidade, estado. Outro exemplo que pode ser citado é referente as informações sobre o incêndio, onde a cor da fumaça possui uma grande importância em indicar o tipo do material que está sendo decomposto no incêndio.

\subsubsection{AVALIAÇÃO DE CONSISTÊNCIA}

Para se avaliar a consistência e é necessário utilizar a analise sintática e semântica, e as regras para se adotar essa avaliação variam de acordo com o contexto que ela está sendo aplicada (Botega, 2016).

Segundo Botega (2016 apud Scannapieco et al., 2005), a inconsistência pode ser exemplificada por um dado em uma resposta provida para um conjunto de dados, no qual o estado civil de uma pessoa é "casado" e a idade do sujeito é de "cinco anos de idade". No contexto deste trabalho a avaliação da consistência será aplicada a partir do segundo alerta referente a mesma situação de incêndio que o sistema receber, e o valor atribuído para alertas consistentes será 0 até $100 \%$. Abaixo são apresentados exemplos de alertas inconsistentes.

Alerta 1: "Há um foco de incêndio próximo a portaria do Jardim Botânico."

Alerta 2: "Acabo de ver fumaça preta próximo a entrada do JBB."

Alerta 3: "Tem uma fumaça rosa no JBB."

Os três alertas foram obtidos pelo aplicativo DF100Fogo para comunidade, os dois primeiros apresentam consistência pois ambos se referem a entrada do JBB, sendo, um informando ter visto fogo e o outro uma fumaça preta, já o terceiro alerta apresenta inconsistência devido a cor da fumaça informado pelo solicitante e por falta de referência em seu alerta. Porém nenhum alerta é descartado, mesmo esse terceiro, para ser validado como um possível trote, é feita a verificação da latitude e longitude do aparelho celular para se ter $100 \%$ de certeza ao não considerar atender essa solicitação.

\subsubsection{AVALIAÇÃO DE CERTEZA DA SIUTAÇÃO}

Ao final da qualificação e quantificação de todas as dimensões e atributos, é feita a avaliação da certeza da situação. Tal dimensão consiste em determinar um índice global para representar a avaliação de qualidade da informação como um todo. Seu cálculo consiste na soma dos resultados de todas as dimensões de qualidade divido pela quantidade de dimensões presentes em cada classe. Tal dimensão incide sobre a confiabilidade que o sistema de informação transmite para o operador humano, Botega (2016) diz que esse cálculo "tratasse de uma generalização preliminar de qualidade calculada em função de cada dimensão de qualidade já calculada", isto pode tornar a tomada de decisão mais segura e assertiva.

\section{ESTUDO DE CASO}


O estudo de caso apresentado nesta seção visa demonstrar o papel da qualidade de dados e informações, em conjunto com a ontologia, como suporte a sistemas críticos e em consequência ajudar os operadores desses sistemas a melhorar sua SAW, promovendo abstrações de alto nível e reduzindo as incertezas no momento da tomada de decisão. Para demonstração de tais resultados, será utilizada a metodologia IQESA, e junto a ontologia o vocabulário DQV para representar as métricas e dimensões de qualidade.

Será abordada uma situação de emergência com incêndio florestal no JBB, neste contexto será necessário entender o cenário da situação e identificar algumas informações relevantes para a resolução do caso como: localização do incêndio, possíveis vítimas, informações sobre o clima e vegetação no local do incêndio.

0 primeiro alerta utilizado para o presente estudo de caso é demostrado na Figura 3 onde já é possível identificar algumas informações que chegam ao sistema dos bombeiros junto com o primeiro alerta feito pelo aplicativo DF100Fogo utilizado pela comunidade local: (1) tipo do alerta, (2) confiabilidade do alerta, esse critério é medido pelo número de alertas verdadeiros que a pessoa já emitiu de seu aplicativo, juntamente com a idade que a pessoa possui, (3) texto do alerta, (4) hora do alerta, o exato momento em que o solicitante enviou o alerta de seu aplicativo, (5) informações sobre o solicitante como nome, idade e localização exata do mesmo no ato do alerta. Detalhes da extração de termos do alerta podem ser obtidos em Botega et al. (2017) e Oliveira et al. (2017).

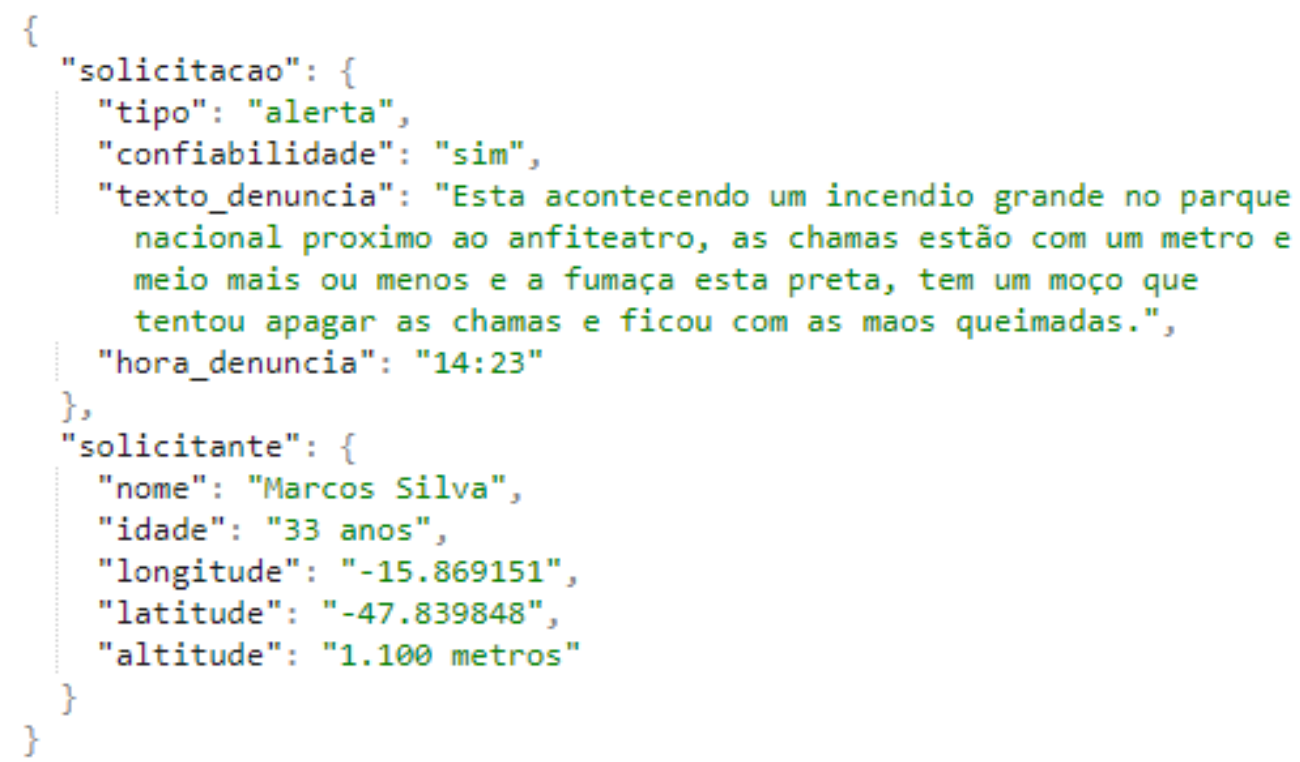

Figura 3: Figura 3: Solicitação de incêndio florestal no JBB em formato JSON6.

Após esse primeiro contato com a solicitação o próprio sistema em um processo de fusão de dados organiza as informações tendo como base a árvore de atributos ilustrada na Figura 1. A partir dessas informações o sistema consegue fazer uso de serviços externos para agregar outras informações ao alerta como por exemplo: dados sobre o clima e topologia no local da ocorrência e algumas informações referente a localização que são buscadas a partir da latitude e longitude da situação de incêndio como por exemplo, bairro, cidade e UF.

0 próximo passo é a utilização da metodologia IQESA para fazer a qualificação e quantificação dos dados e informações junto com as dimensões de Completude, Relevâncias, Atualidade e Consistência.

\footnotetext{
${ }^{6}$ Formato compacto, de troca de dados simples e rápida entre sistemas https://www.json.org/json-pt.html
} 
Quadro 1: Avaliações de Qualidade do Primeiro Alerta

\begin{tabular}{|l|c|c|c|c|c|}
\hline \multicolumn{1}{|c|}{ Entidades } & Completude & Relevância & Atualidade & Consistência & Certeza \\
\hline Local & $82 \%$ & 6 & nulo & nulo & nulo \\
\hline Terreno & $100 \%$ & 1 & nulo & nulo & nulo \\
\hline Patrimônio & $69,4 \%$ & 3 & nulo & nulo & nulo \\
\hline Fogo & $39,7 \%$ & 2 & nulo & nulo & nulo \\
\hline Superfície & $100 \%$ & 1 & nulo & nulo & nulo \\
\hline Fumaça & $69,4 \%$ & 1 & nulo & nulo & nulo \\
\hline Clima & $100 \%$ & 7 & nulo & nulo & nulo \\
\hline Vítima & $39,7 \%$ & 3 & nulo & nulo & nulo \\
\hline Solicitante & $100 \%$ & 2 & nulo & nulo & nulo \\
\hline Solicitação & $100 \%$ & 3 & 2 minutos & nulo & $\mathbf{3 9 , 5 3 \%}$ \\
\hline
\end{tabular}

Fonte: Elaborado pelos autores

A dimensão de Consistência só é calculada a partir do segundo alerta sobre a mesma situação de incêndio. 0 cálculo da Certeza se dá pela somatória de todos os índices calculados $(830,2)$, dividido pela quantidade de índices presentes no alerta (29) resultando em 39,53\%. Os índices de Completudes, Relevância e Atualidade são calculados conforme formulas demonstradas nas seções anteriores:

- Local: dos 12 atributos presentes na classe, apenas 10 possuem valores, aplicando esses valores a fórmula de completude o índice encontrado é de $82 \%$. Dos 10 atributos presentes somente 6 são relevantes.

- Terreno: possui apenas um atributo com valor sendo o índice de completude de $100 \%$. Possui valor 1 de relevância.

- Patrimônio: possui 6 atributos desses 4 possuem valores sendo o índice de completude de 69,4\%. Possui 3 valores relevantes.

- Fogo: possui 6 atributos, desses apenas 2 possui valor gerando um índice de completude de 39,7\%. Possui 2 valores relevantes.

- Superfície: tipo do incêndio identificado no local, possui 1 atributo e o mesmo tem valor, gerando uma completude de $100 \%$. Possui 1 atributo relevante.

- Fumaça: possui 2 atributos, sendo somente $1 \mathrm{com}$ valor gerando um índice de completude de 69,4\%. Possui 1 atributo relevantes.

- Clima: possui 8 atributos todos com valores presentes, gerando um índice de completude de $100 \%$. Possui 7 atributos relevantes.

- Vítima: possui 9 atributos, desses somente 3 estão completos gerando um índice de completude de 39,7\%. Possui 3 atributos relevantes.

- Solicitante: possui 5 atributos todos completos gerando uma completude de 100\%. Possui 2 atributos relevantes.

- Solicitação: possui 4 atributos, todos com valores gerando um índice de completude de $100 \%$. Possui 3 atributos relevantes. Para cálculo da Atualidade foi subtraído o horário da solicitação, $14 \mathrm{~h} 23$ do horário do sistema quando recebeu o alerta as $14 \mathrm{~h} 25$, gerando um valor de atualidade de 2 minutos.

0 segundo alerta entregue ao sistema foi enviado pelo aplicativo DF100Fogo utilizado pelos bombeiros.

Alerta 2: "Grande incêndio registrado próximo ao anfiteatro em vegetação tipo mata de galeria na unidade de conservação, possui vítima no local com olhos ardendo, mãos queimadas e dificuldades para respirar, feito na vítima primeiros atendimentos, área isolada de 95 metros, intensidade do fogo de $500 \mathrm{Kcal} / \mathrm{m}$ e taxa de alastramento de $2 \mathrm{~m} / \mathrm{s}$, com fumaça preta de mais ou menos $5 \mathrm{~m}$ e chamas de 1,5 metros".

É possível verificar que este alerta é mais técnico e completo pois se trata de um bombeiro de campo que verificou a situação pelo aplicativo do DF100Fogo para bombeiros e foi até o local verificar a real situação de incêndio. A cada nova informação referente a mesma situação que o sistema recebe o mesmo processo para avaliação de qualidade de 
dados ocorre. 0 Quadro 2 apresenta os novos resultados referente as dimensões de Certeza, Completude, Consistência, Atualidade e Relevância para a situação.

Quadro 2: Avaliações de Qualidade do Segundo Alerta

\begin{tabular}{|c|c|c|c|c|c|}
\hline Entidades & Completude & Relevância & Atualidade & Consistência & Certeza \\
\hline Local & $46 \%$ & 4 & nulo & nulo & nulo \\
\hline Terreno & $100 \%$ & 1 & nulo & nulo & nulo \\
\hline Vegetação & $100 \%$ & 2 & nulo & nulo & nulo \\
\hline Patrimônio & $100 \%$ & 5 & nulo & nulo & nulo \\
\hline Isolamento & $100 \%$ & 4 & nulo & nulo & nulo \\
\hline Vítima & $69,4 \%$ & 6 & nulo & nulo & nulo \\
\hline Solicitante & $100 \%$ & 2 & nulo & nulo & nulo \\
\hline Bombeiro & $100 \%$ & 5 & nulo & nulo & nulo \\
\hline Fogo & $100 \%$ & 6 & nulo & nulo & nulo \\
\hline Superfície & $100 \%$ & 1 & nulo & nulo & nulo \\
\hline Fumaça & $100 \%$ & 1 & nulo & nulo & nulo \\
\hline Recursos Alocados & $100 \%$ & 1 & 3 minutos & $100 \%$ & $48,54 \%$ \\
\hline Solicitação & $100 \%$ & 3 & & & nulo \\
\hline
\end{tabular}

Fonte: Elaborado pelos autores

No Quadro 2 é possível ver o cálculo da consistência, essa dimensão é aplicada a partir de novas informações processadas para uma situação que já está em curso, para se aplicar a métrica dessa dimensão é necessário se ter uma base de informações para a comparação com as novas que chegam ao sistema. 0 cálculo resultou em $100 \%$ pois os dois alertas continham informações sobre o mesmo incêndio, algumas até se repetiram confirmando assim a sua consistência. 0 cálculo da dimensão certeza foi de 48,54\%, pois as novas informações ajudaram a melhorar os índices de qualidade das demais dimensões. 0 Quadro 3 ilustra os valores de qualidade medidos para a fusão dos alertas 1 e 2, o processo para esses cálculos foram os mesmos já demostrados anteriormente.

Após esses cálculos sobre a qualidade, a ontologia instanciada será demonstrada na Figura 4. Como já conceituada a ontologia é um mecanismo de representação de dados e informações, ao ser instanciada com os dados presentes nos dois alertas a ontologia poderá apresentar novas relações entre as entidades, esse tipo de processo contribui de forma positiva na inferência de conhecimento para o humano operador de sistemas críticos.

A Figura 5 demonstra parte dos dados gerados em formato JSON-LD, tais dados serão enviados aos serviços de interfaces. A partir desse passo é demostrado ao humano operador, novas relações e informações sobre o alerta, subsidiando assim uma tomada de decisão mais assertiva e confiável, além de contribuir com a SAW de tal operador.

Quadro 3: Avaliações de Qualidade da Fusão dos Alertas 1 e 2

\begin{tabular}{|c|c|c|c|c|c|}
\hline Entidades & Completude & Relevância & Atualidade & Consistência & Certeza \\
\hline Local & $82 \%$ & 6 & nulo & nulo & nulo \\
\hline Terreno & $100 \%$ & 1 & nulo & nulo & nulo \\
\hline Vegetação & $100 \%$ & 2 & nulo & nulo & nulo \\
\hline Patrimônio & $100 \%$ & 5 & nulo & nulo & nulo \\
\hline Isolamento & $100 \%$ & 4 & nulo & nulo & nulo \\
\hline Clima & $100 \%$ & 7 & nulo & nulo & nulo \\
\hline Vítima & $69,4 \%$ & 6 & nulo & nulo & nulo \\
\hline Solicitante & $100 \%$ & 2 & nulo & nulo & nulo \\
\hline Bombeiro & $100 \%$ & 5 & nulo & nulo & nulo \\
\hline Fogo & $100 \%$ & 6 & nulo & nulo & nulo \\
\hline Superfície & $100 \%$ & 1 & nulo & nulo & nulo \\
\hline Fumaça & $100 \%$ & 1 & nulo & nulo & nulo \\
\hline Recursos locados & $100 \%$ & 1 & nulo & nulo & nulo \\
\hline Solicitação_1 & $100 \%$ & 3 & 2 minutos & nulo & nulo \\
\hline Solicitação_2 & $100 \%$ & 3 & 3 minutos & $100 \%$ & \\
\hline & & & 5 minutos & $100 \%$ & $48,77 \%$ \\
\hline
\end{tabular}




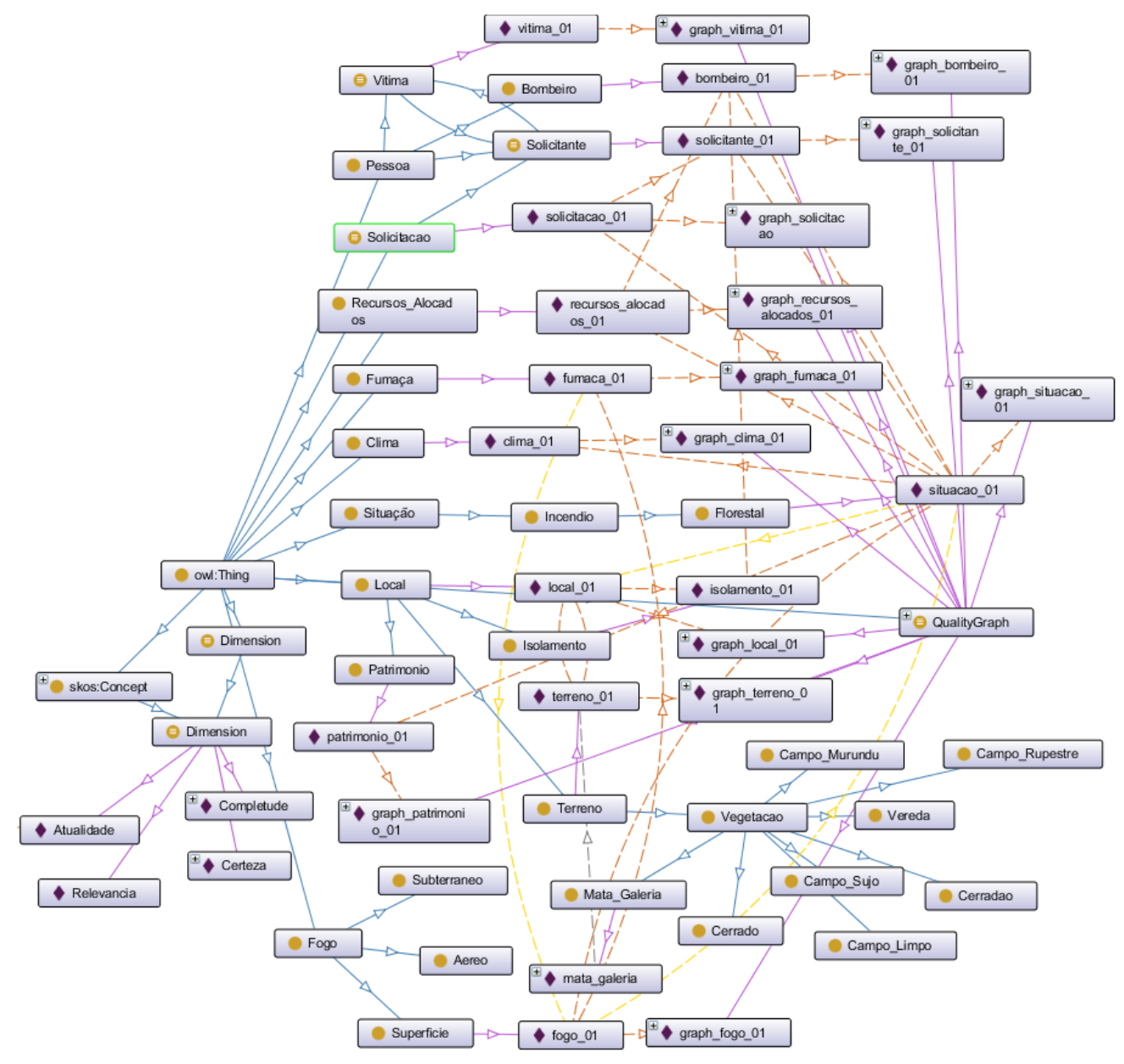

Figura 4: Ontologia gerada a partir dos dados dos Alertas 1 e 2. Fonte: Elaborado pelos autores

Para validar a ontologia demonstrada na Figura 5, foram utilizadas consultas SPARQL, essas consultas permitem ao operador de sistemas de emergências uma melhor inferência sobre a situação de incêndio, produzindo assim mais certeza para uma tomada de decisão mais assertiva. Considere os prefixos demonstrados no Código Fonte 1 para todas as consultas apresentadas ao longo desta sessão.

A primeira consulta (Código Fonte 2) executada demonstra informações sobre a Classe Local, informações referentes a latitude e longitude são de extrema importância no domínio de incêndio florestal, pois a partir dessa localização foi possível verificar que a situação de incêndio ilustrada abaixo está acontecendo em uma unidade de conservação.

A segunda consulta feita através do Código Fonte 3 e demonstrada no Quadro 5 também foi efetuada na classe Local, porém agora é possível verificar os valores referente as dimensões de Completude e Relevância, providos da qualificação dos dados. As instâncias Graph, Qt, Qt_value e Métrica são do vocabulário DQV e demonstram resultado de 84,7\% para Completude e 9 de Relevância, ou para o operador as informações referentes ao local do incêndio possuem significância e integridade alta, o que prove a tal operador uma segurança na tomada de decisão. 


\section{\{}

"@context": \{

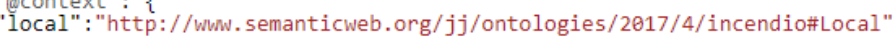

"terreno": "http://wWl. semanticweb.org/jj/ontologies/2017/4/incendio\#terreno",

"vegetaçăo": http://WWh.semanticweb.org/jj/ontologies/2017/4/incendio\#Vegetacao",

"patrimonio": "http://WWl.semanticweb.org/jj/ontologies/2017/4/incendio\#Patrimonio",

"isolamento": "http://wWl.semanticweb.org/jj/ontologies/2017/4/incendio\#Isolamento",

"solicitacao": "http://Wlw. semanticweb.org/jj/ontologies/2017/4/incendio\#Solicitacao",

"clima": "http://wWw.semanticweb.org/jj/ontologies/2017/4/incendio\#Clima" \} , \}

"@graph": [

"@id": "http://www.semanticweb.org/jj/ontologies/2017/4/incendio\#bombeiro_01",

"@type":

http:// / wnww.w3.org/2002/07/owl\#NamedIndividual",

"hittp://www. semanticweb.org/jj/ontologies/2017/4/incendio\#latitude": \{ \}, 1 "Qtype": "http://WwW.W3.org/2001/XMLSchema\#decimal",

"http://www.semanticweb.org/jj/ontologies/2017/4/incendio\#longitude" : \{ "@type": "http:// www.w3.org/2001/XMLSchema\#decimal",

"http://www.semanticweb.org/jj/ontologies/2017/4/incendio\#nome": "José Antonio da Silva",

"http://www. semanticweb.org/Yj/ontologies/2017/4/incendio\#patente": "brigadista",

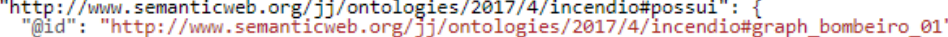

"http://ww semanticweb.org/ji/ontologies/2017/4/incendio\#responsabilidade": "verificar denuncia e isolar a area", "http://www. semanticweb.org//j/ontologies/2017/4/incendio\#viatura": \}$^{\}}$

"@type": "http://www.w3.org/2001/XMLSchema\#integer",

\}$^{]}$ ]$^{\}}$

Figura 5: Parte do JSON-LD referente à Ontologia.

Fonte: Elaborado pelos autores

PREFIX rdf: 〈http://WWl.w3.org/1999/02/22-rdf-syntax-ns\#〉

PREFIX rdfs: 〈http://Whlw.w3.org/2000/01/rdf-schema\#〉

PREFIX owl: 〈http://Wwwlw3.org/2002/07/owl\#〉

PREFIX xmls: 〈http://Ww/w.w3.org/2001/XMLSchema\#〉

PREFIX dqv: 〈http://WWwl.w3.org/ns/dqv\#>

PREFIX incendio: 〈http://WWW.semanticweb.org/jj/ontologies/2017/4/incendio\#>

Código Fonte 1 Prefixos utilizados para as consultas SPARQL

Fonte: Elaborado pelos autores

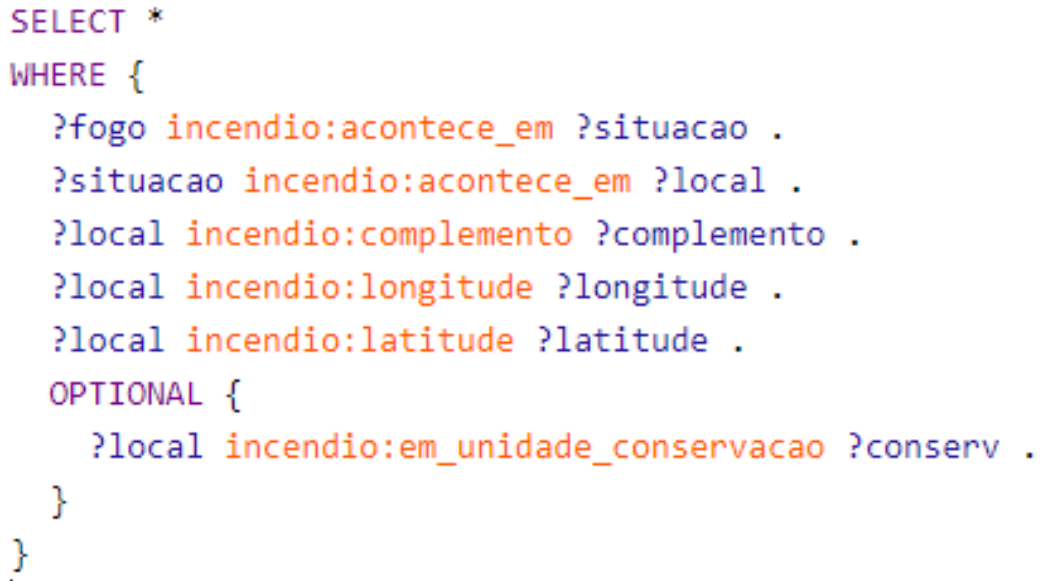

Código Fonte 1: Consulta SPARQL Classe Local Fonte: Elaborado pelos autores 
Quadro 4: Resultado da Consulta SPARQL para a classe Local

\begin{tabular}{|l|l|}
\hline Fogo & Incendio:fogo_01 \\
\hline Situação & Incendio:situação_01 \\
\hline Local & Incendio:local_01 \\
\hline Complemento & "Próximo ao Anfiteatro" \\
\hline Latitude & -15.868840 \\
\hline Longitude & -47.839613 \\
\hline Conservação & true \\
\hline
\end{tabular}

Fonte: Elaborado pelos autores

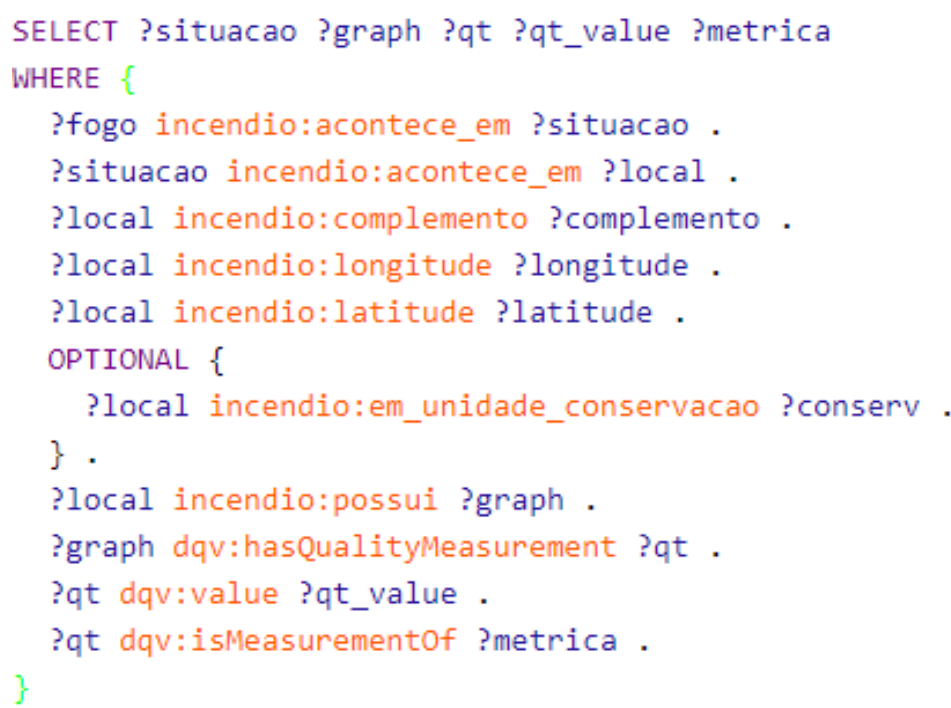

Código Fonte 2: Consulta SPARQL Classe Local com Qualificação de dados Fonte: Elaborado pelos autores

Quadro 5: Resultado da Consulta SPARQL para a classe Local com Qualificação de Dados

\begin{tabular}{|l|c|c|}
\hline & Completude & Relevância \\
\hline Situação & Incendio:situação_01 & Incendio:situação_01 \\
\hline Graph & Incendio:graph_local_01 & Incendio:graph_local_01 \\
\hline Qt & Incendio:qt_local_comp_01 & Incendio:qt_local_relev_01 \\
\hline Qt_value & $84,7 \%$ & 9 \\
\hline Métrica & Incendio:MetricaLocalCompletude & Incendio:MetricaLocalRelevancia \\
\hline
\end{tabular}

Fonte: Elaborado pelos autores

A terceira consulta demostra o relacionamento entre as Classe Bombeiro e Isolamento, na ontologia o bombeiro responsável por verificar a situação de emergência, também fica responsável por isolar a área.

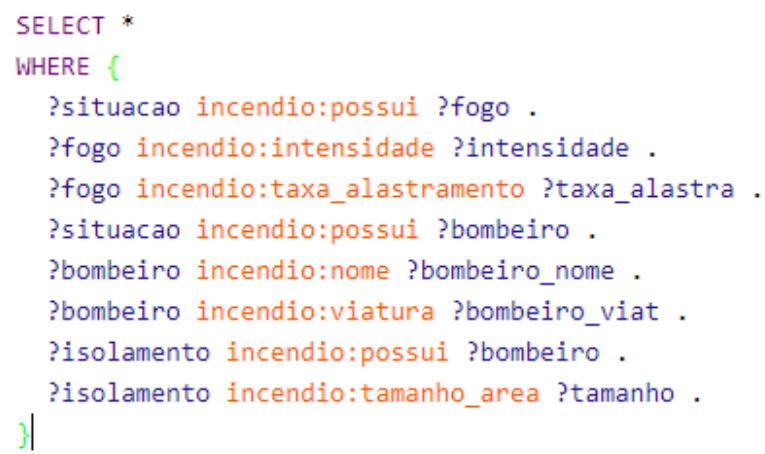

Código Fonte 3: Consulta SPARQL Relação entre as Classes Bombeiro e Isolamento Fonte: Elaborado pelos autores 
Quadro 6: Resultado da Consulta SPARQL para a Relação entre as classes Bombeiro e Isolamento

Fonte: Elaborado pelos autores

\begin{tabular}{|l|l|}
\hline Situação & Incendio:situação_01 \\
\hline Fogo & Incendio:fogo_01 \\
\hline Intensidade & $500 \mathrm{Kcal} / \mathrm{m}$ \\
\hline Taxa_alastramento & $2 \mathrm{~m} / \mathrm{s}$ \\
\hline Bombeiro & Incensio:bombeiro_01 \\
\hline Bombeiro_nome & "Jose Antônio da Silva" \\
\hline Bombeiro_viatura & 12 \\
\hline Isolamento & Incendio:isolamento_01 \\
\hline Tamanho & 95 metros \\
\hline
\end{tabular}

\section{CONCLUSÕES}

O presente trabalho teve como objetivo o desenvolvimento de uma ontologia ciente de qualidade de dados, visando contribuir com a aquisição e melhora nos níveis de SAW em operadores humanos de sistemas de emergência. No contexto de Ciência da Informação, este trabalho visou demonstrar que o uso de informações qualificadas e quantificadas auxiliam sistemas e humanos na tomada de decisão, pois existe uma maior segurança e integridade nas informações obtidas devido a ontologia utilizada e a gestão de qualidade empregada uma para quantificar e qualificar os dados processados.

A ontologia poderá proporcionar ao sistema DF100Fogo a representação de informações mais clara e objetiva dentro do domínio de gerenciamento de emergência através de ilustrações de todas as entidades presentes em uma situação de emergência e suas possíveis relações, até mesmo fazendo inferência de novos dados sobre determinada situação.

Em sistemas de domínio emergência é comum encontrar relações não explicitas entre as entidades, o que pode ser resolvido com o uso de ontologias, além disso, o auxilio se estende aos operadores humanos, também contribuindo positivamente com aquisição e manutenção de sua SAW, pois a adoção de qualificação dos dados junto a ontologia faz com que as informações consumidas pelos operadores em um atendimento a emergência sejam mais concisas e precisas tornando assim as tomada de decisão por parte do operador mais segura e assertiva.

Para ilustrar os resultados obtidos neste trabalho foi desenvolvido e apresentado um caso, no qual pode-se constatar em um primeiro momento que os resultados provenientes das consultas realizadas junto aos dados da ontologia apresentada demonstraram que as relações entre as diversas entidades presentes em um atendimento a emergência podem se tornar explicitas para o operador, subsidiando assim um conhecimento amplo a situação.

Também é importante ressaltar que o domínio apresentando neste trabalho traz consigo muitas incertezas o que gera para a área de qualidade e sistemas de apoio a decisão grandes desafios. Para trabalhos futuros tal ontologia será finalizada e incorporada ao sistema de avalição de situações de emergência o DF100Fogo.

\section{REFERÊNCIAS}

AMAILEF, K., LU, J. Ontology-supported case-based reasoning approach for intelligent m-Government emergency response services. Decision Support Systems, v. 55, n. 1, p. 79-97, 2013. Disponível em: <https://doi.org/10.1016/j.dss.2012.12.034>. Acesso em: 30 jun. 2018.

BOTEGA, L. C., VALDIR, A. P., OLIVEIRA, A. C., SARAN, J. F., VILLAS, L. A., DE ARAÚJO, R. B. Quality-aware human-driven information fusion model. In: INTERNATIONAL CONFERENCE ON INFORMATION FUSION, 20, 2017. Anais eletrônicos... p. 1-10, 2017. Disponível em: <https://ieeexplore.ieee.org/abstract/document/8009851/>. Acesso em: 30 jun. 2018.

BOTEGA, L. C., SOUZA, J. O., JORGE, F. R., CONEGLIAN, C. S., CAMPOS, M. R., ALMEIDA NERIS, V. P., ARAÚJO, R. B. Methodology for Data and Information Quality Assessment in the Context of Emergency Situational Awareness. Universal Access in the Information Society, v. 16, n. 4, p. 889-902. Disponível em: <https://link.springer.com/content/pdf/10.1007\%2Fs10209-016-0473-0.pdf>. Acesso em: 30 jun. 2018.

ENDSLEY, M. R. Designing for Situation Awareness in Complex System. In: PROCEEDINGS OF THE INTERNATIONAL WORKSHOP ON SYMBIOSIS OF HUMANS, ARTIFACTS AND ENVIRONMENT, 2, 2001. 
Anais $\quad$ eletrônicos...
<https://www.researchgate.net/profile/Mica_Endsley/publication/238653506_Designing_for_situatio
n_awareness_in_complex_system/links/542b1ada0cf29bbc126a7f35.pdf $>$. Acesso em: 30 jun. 2018 .

ENDSLEY, M. R., BOLSTAD, C. A., JONES, D. G., RILEY, J. M. Situation awareness oriented design: from user's cognitive requirements to creating effective supporting technologies. In: HUMAN FACTORS AND ERGONOMICS SOCIETY ANNUAL MEETING, v. 47, n. 3, 2003. Anais eletrônicos... Sage CA: Los Angeles, CA: SAGE Publications, 2003, p. 268-272. Disponível em: <http://journals.sagepub.com/doi/pdf/10.1177/154193120304700304>. Acesso em: 30 jun. 2018.

LI, X., LIU, G., LING, A., ZHAN, J., AN, N., LI, L., SHA, Y. Building a practical ontology for emergency response systems. In: INTERNATIONAL CONFERENCE ON COMPUTER SCIENCE AND SOFTWARE ENGINEERING, 4, 2008. Anais eletrônicos... p. 222-225. Disponível em: <https://ieeexplore.ieee.org/abstract/document/4722603/>. Acesso em: 30 jun. 2018.

MELO, J. O. Metodologia de Avaliação de Qualidade de Dados no Contexto do Linked Data Dissertação (Mestrado em Ciência da Informação) - Universidade Estadual Paulista "Júlio Mesquita Filho", Faculdade de Filosofia e Ciências, Marília, 2017. Disponível em: <https://repositorio.unesp.br/bitstream/handle/11449/150870/melo_josf_me_mar.pdf?sequence=3\& isAllowed=y>. Acesso em: 30 jun. 2018.

NOY, N. F., McGUINNESS, D. L. Ontology Development 101: A guide to creating your first ontology. Stanford Knowledge Systems Laboratory Technical Report KSL-01-05 and Stanford Medical Informatics Technical Report SMI-2001-0880, 2001. Disponível em: $<$ https://protege.stanford.edu/publications/ontology_development/ontology101.pdf >. Acesso em: 30 jun. 2018.

OLIVEIRA, A. C., BOTEGA, L. C., SARAN, J. F., bbbbSILVA, J. N., MELO, J. O., TAVARES, M. F., NERIS, V. P. Crowdsourcing, data and information fusion and situation awareness for emergency management of forest fires: the project DF100Fogo (DFWithoutFire). Computers, Environment and Urban Systems, 2017. Disponível em: <https://doi.org/10.1016/j.compenvurbsys.2017.08.006 >. Acesso em: 30 jun. 2018.

SOUZA, A., CORCHO, O., SALLES, P., VILCHES-BLÁZQUEZ, L. An application of geographical and statistical linked data to ecology: the Brazilian cerrado ontology network and qualitative reasoning models. In: INTERNATIONAL CONGRESS ON ENVIRONMENTAL MODELLING AND SOFTWARE SOCIETY, 7, San Diego, 2014. Anais eletrônicos... p. 362-369, 2014. Disponível em: $<$ https://scholarsarchive.byu.edu/cgi/viewcontent.cgi?article=1045\&context=iemssconference $>$. Acesso em: 30 jun. 2018.

Editores do artigo: Enrique Muriel-Torrado, Edgar Bisset Alvarez, Camila Barros. 